\title{
First Option for Fusion with CPA-Laser Pulses instead of Thermal Pressures with Dozens of Million Degrees Temperature for Laser-Boron-Fusion
}

\author{
Heinrich Hora ${ }^{1,2}$, Götz J. Kirchhoff ${ }^{2}$, Warren R. McKenzie ${ }^{2}$ and Jan Kirchhoff ${ }^{2}$ \\ 1. Department of Theoretical Physics, University of NSW, Sydney 2096, Australia \\ 2. HB11 Energy Pty. Ltd, Freshwater, Sydney 2096, Australia
}

\begin{abstract}
To avoid the $>50$ million degree temperatures in pressures for fusion, the goal has now been reached that higher pressures from picosecond CPA-laser pulses of more than $10 \mathrm{PW}$ power can ignite nuclear fusion at modest temperatures in a power reactor for generating electricity. Gerard Mourou, who was honoured with the 2018 Physics Nobel Prize, was involved to solve this crucial problem of fusion energy with the reactor design as co-authors (Hora, H., Korn, G., Giuffrida, L., Margarone, D., Picciotto, A., Krasa, J., Jungwirth, K., Ullschmied, J., Lalousis, P., Eliezer, S., Miley, G. H., Moustaizis, S., and Mourou, G., Laser and Particle Beams 33, 2015: 607) using the CPA Chirped Pulse Amplification.
\end{abstract}

Key words: Ignition of nuclear fusion, clean nuclear power stations, ultra-extreme CPA-laser pulses.

\section{Introduction}

The modern wealth of living is based on energy mostly from chemical reactions including the burning of carbon. This needs now a drastic limitation to reduce the emission of carbon-dioxide to $20 \%$ from the present level to solve the energy crisis. One alternative to the chemical energy is to use nuclear energy that is ten million times larger per reaction and is available from uranium fission from which the verified use for generators produces more than $10 \%$ of all of the present global demand.

An alternative to gain nuclear energy uses nuclear fusion aiming to eliminate the problems of nuclear radiation in contrast to nuclear fission. Fusion energy is the key energy source in the stars of the universe. Expensive and most sophisticated research is done for a generator of electricity since 65 years but not yet available. The easiest reaction is to burn heavy

Corresponding author: Prof. Heinrich Hora, Dr.rer.nat., D.Sc., research fields: nonlinear, relativistic and quantum effects of laser-interaction. hydrogen D with superheavy $\mathrm{T}$, respectively known as deuterium with tritium, as DT-reaction. Interesting is also the fusion of ordinary hydrogen $\mathrm{H}$ with the abundant isotope 11 of boron (HB11 reaction), because the burning is primarily absolute clean without producing any dangerous radioactive ash. The product is harmless helium, known also as alpha particles. A problem with a minor contribution of secondary reactions can be under control below the tolerable limit.

Why is nuclear fusion so difficult? The key problem for controlled generation fusion in a reactor for electricity using the usual hydrodynamic pressures, is the needed temperature of more than 50 millions degrees Celsius. The sixty-five years of research has well achieved these temperatures under conditions in laboratories, both for ITER-like magnetic confinement as well as for NIF-like inertial confinement fusion ICF when using nanosecond laser pulses. The temperatures were produced, but for too short times such that a breakthrough for a reactor has not yet been reached. 
The need for million degrees temperatures is no surprise. The energy from chemical reactions - as the burning of carbon - is in the range of electron volts, $\mathrm{eV}$, needing ignition temperatures of few hundred of degrees Kelvin like ignition by a match. The ten million times higher energies for nuclear reactions need then dozens of million degrees under thermal pressures. The sun burns hydrogen to helium at 15 million degrees and the first deuterium fusion reactions at thermal equilibrium at magnetic confinement in a stellarator were measured in 1980 at 10 million degrees [1].

\section{Lasers Offer a Solution}

When the laser was discovered in 1960, it was obvious for physicists from the level of the highest celebrities, Edward Teller and Andrei Sakharov that this is radically changing to nonlinear physics results in exploring "inertial confinement fusion and high energy density science" [2] to solve the generation of electricity from the power of nuclear fusion. Extremely high energy densities of laser pulses were expected to be concentrated within extremely short times into extremely small volumes by the laser. This is related to nonlinear physics from the very early beginning.

Firing laser pulses of less than MW power on targets by laser pulses of about $10 \mathrm{~ns}$ duration on solid targets in vacuum showed classical properties. The pulse was absorbed in the focus at the target and the temperature of few dozens of ten thousand degrees Kelvin resulted in fully classical emission of ions of several $\mathrm{eV}$ energy. When the laser pulse has not very much higher than MW power, suddenly, ions of up to thousand times higher energy $\mathrm{keV}$ were measured [3]. However, these ions did not show a thermal origin with equally distributed thermal energy, but the ions were in groups separated linear on the ion charge indicating a non-thermal electro-dynamic origin.

The same was observed from the emission of electrons [4] where emission current densities did not follow the classical Langmuir-Child law but were increased by few orders of magnitudes. The energies of the fast ions increased on the square of the laser intensity [5] while thermal models resulted in exponents of about 0.3 and not 2 [5].

The highly nonlinear property was seen from side-on photos of laser irradiated aluminium spheres $[6,7]$, in Fig. 1, where a spherical core expanded according to classical thermal expansion and a faster half-moon like plasma moved on nearly a square dependence of the laser intensity showing the nonlinear interaction property.

A first step to understand the nonlinear interaction different from thermal properties was to consider a collsionless plasma slab with a density profile and a related optical refraction of Fig. 2. The hydrodynamic forces given by the arrows are driving the plasma each

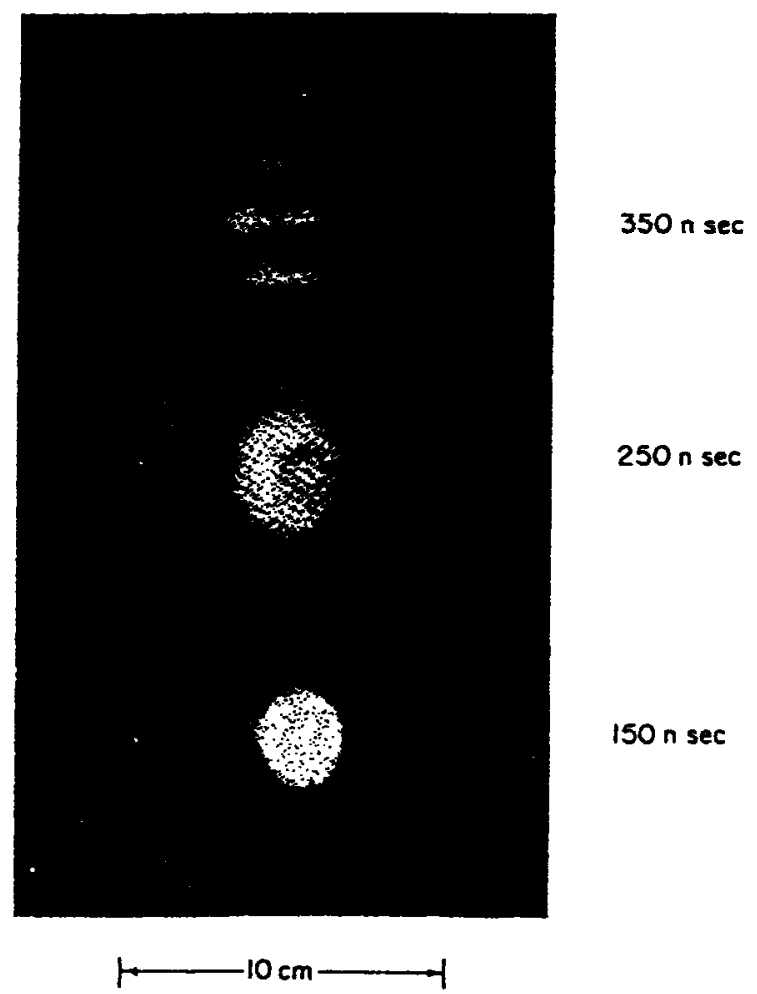

Fig. 1 Side-on framing camera picture of a plasma produced from an aluminum sphere of $80 \mu \mathrm{m}$ radius at the time indicated after irradiation by a $30 \mathrm{~ns}$ ruby laser pulse focused to $0.4 \mathrm{~mm}$ diameter at Westinghouse research [6, 7] (Fig. 1.4 of Ref. [8]). The second frame shows the outer part of a rapidly expanding plasma and an inner spherical thermally expanding part. 


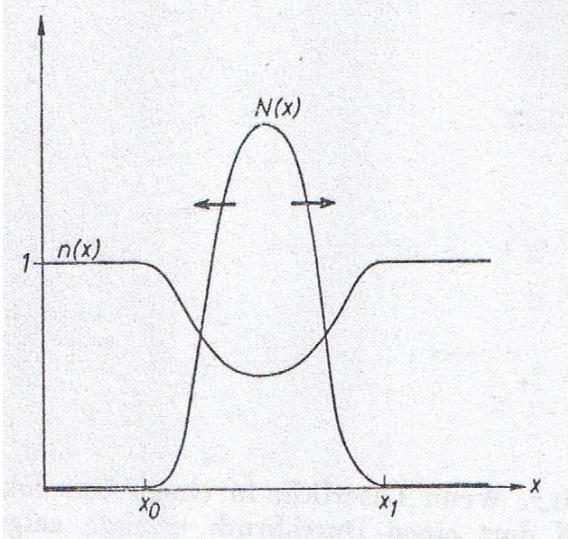

Fig. 2 A laser pulse arriving from the left hand side on a plasma slab of density $N(x)$ with the schematic drawn curve for the optical constant $n(x)$ within the plasma decreasing below the value unity, is causing nonlinear (ponderomotive) forces (arrows) in the plasma equation of motion tearing the slap as a dielectric explosion into a part moving against and another with the laser beam [9].

towards the vacuum as a kind of a dielectric explosion process. This can be basically understood from theory of plasma motion of a slab of collisionless plasma with inhomogeneous optical refraction resulting in forces of a dielectric explosion, in Fig. 2 [9].

For realistic conditions of plasmas with thermal properties and heating, the general equations of motions were needed. Missing nonlinear terms in the plasma hydrodynamic equations of motion had to be added [10] from conservation of the momentum when including the optical constants of the plasma. The equation of motion for the force density $f$ has then a term with the thermal gas-dynamic pressure $p$ given by density and temperature $T$.

$$
f=-\nabla p+f_{\mathrm{NL}}
$$

and a second term of the nonlinear force $f_{\mathrm{NL}}$ caused by electric and magnetic laser fields $E$ and $H$, the plasma frequency $\omega$ and the optical refractive index $n$. This is given as $f_{\mathrm{NL}}=\nabla \bullet M$ with Maxwell's stress tensor $M$.

$$
M=\left[E E+H H-0.5\left(E^{2}+H^{2}\right) 1+\right.
$$

$$
\left.(1+(\partial / \partial t) / \omega)\left(n^{2}-1\right) E E\right] /(4 \pi)-(\partial / \partial t) E \times H /(4 \pi c)
$$

These forces are nonlinear, because they relate mechanical force density with the squares of force quantities $E$ and $H$. This is gauge and Lorentz invariant for the non-transient case and is connected to the plasma dynamical case $[11,12]$.

One key application of the laser is the question, how and when the second term of Eq. (1) can be larger against the first term. The question is then that the non-thermal pressure by the nonlinear force from laser pulses becomes larger and dominates against the thermal pressure of the first term. This is then the key question that the non-thermal nonlinear force can drive fusion reactions without needing thermal pressures with temperatures of hundred million degrees. On the way to this exploration, a large number of facts had to be discovered. Last not least it happened that the evaluation of nonlinear forces could arrive in wrong predictions in linear physics and only nonlinear physics with inclusion of very tiny neglected parameters could lead to the correct results in nonlinear physics (see Feynman [13]).

As an example, the numerical evaluation of realistic plasma with general hydrodynamics and collisional heating was shown in 1977, Fig. 3 (from Figs. 10.18a \& 10.18b of Ref. [8], drawn together in Fig. 8.4 of Ref. [12]) where the second nonlinear term in Eq. (1) dominates over the thermal pressure term.

Using Chirped Pulse Amplified (CPA) laser pulses [14] with parameters close to the conditions of the computations of Fig. 3, Sauerbrey [15] measured exactly the superhigh acceleration of $2 \times 10^{20} \mathrm{~cm} / \mathrm{s}^{2}$ of the plasma block moving against the incident laser pulse, seen from the blue Doppler shift of spectral lines. This acceleration was about 100,000 times higher than equivalent thermal acceleration using the biggest laser with nanosecond pulses. The basically different measurements of nonlinearities against the usual linear observations were measured by Badziak et al. [16] and clarified [17] to be basically different from the usual results of thermal equilibrium conditions including relativistic self-focusing. The difference was seen from the energy of the fastest moving plasma against the laser pulse to be increasing on the square of the incident laser pulses. Indeed it was necessary that the picosecong laser pulses had to be of exceptional 


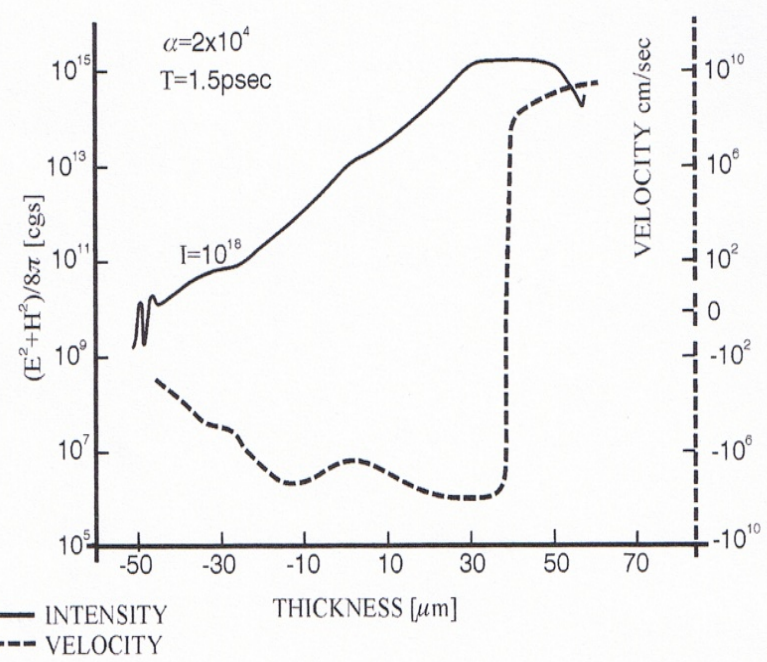

Fig. $310^{18} \mathrm{~W} / \mathrm{cm}^{2}$ neodymium glass laser is incident from the right hand side on an initially $100 \mathrm{eV}$ hot $100 \mu \mathrm{m}$ deuterium plasma slab with initially low reflecting bi-Rayleigh density profile at initial time $t=0$, resulting at time $t=1.5 \mathrm{ps}$ of interaction in a velocity distribution $v(x)$ on the depth $x$ and in an energy density of the laser field $\left(E^{2}+H^{2}\right) / 8 \pi$. The dynamic development had accelerated the plasma block of about 20 vacuum wave length thickness of the skin layer moving against the laser and another block into the plasma showing ultrahigh $>10^{20} \mathrm{~cm} / \mathrm{s}^{2}$ dielectric acceleration by the nonlinear force.

quality with respect to the contrast number in Refs. $[15,16]$ as a crucial precondition. This was demonstrated in the experiments of the team of Jie Zhang et al. [18] from the need that relativistic self-focussing [19] had to suppress.

It was essential for the non-thermal, nonlinear force driven plasma block acceleration of Fig. 3, that a picosecond interaction process of the CPA laser process with powers up to the petawatt range was happening. Without having the result of the plasma block mechanism, an unspecified ps initiation of the ignition of DT-fusion in solid density targets [20] was calculated that an energy flux density above a threshold $\mathrm{E}^{*}$ of $4 \times 10^{8} \mathrm{~J} / \mathrm{cm}^{2}$ for an interaction time of one picosecond was necessary. After updating of these [20] calculations by later known properties of laser-plasma interaction [21] a surprising result was derived. For DT fusion, a threshold for the energy flux density $\mathrm{E}^{*}$ of $4 \times 10^{8} \mathrm{~W} / \mathrm{cm}^{2}$ for the ps initiation of the ignition was needed. What was surprising was that the plasma block ignition of HB11 fusion [22] arrived at nearly the same threshold $\mathrm{E}^{*}$ as for DT. In contrast, when comparing this with the HB11 fusion at equilibrium thermal conditions, it was well known that the energy gain resulted in five orders of magnitudes lower value than DT (see Chapter 9.6 of Ref. [12]). It was therefore very unexpected that the threshold E* for HB11 was on a similar level $[22,23]$ close to that for DT fusion, jumping over the five orders of magnitudes in the energy gains. This calculation of five orders of magnitudes improvement was even pessimistically using only binary fusion reactions for comparison [23].

\section{Experiments with Dominating Non-thermal Plasma Block Acceleration}

Another experimental example for plasma block initiation is given with the experiments by Norreys et al. [24] where the fusion energy gain of DD reactions was increased by a factor of about 10,000 in contrast to the thermal pressure driving. This was realized in retrospect only by the here described developments. The consideration was initiated by Krasa et al. [25] following an evaluation of a large number of various experiments on neutron emission from fusion of deuterium containing solid density targets at differing times of irradiated laser pulses, in Fig. 4. For pulse length of a picoseconds, the neutron gain was 10,000 times higher than the measurements with reactions under all the numerous results with thermal equilibrium conditions. It was confirmed by the measurements [24] that the temperature of the four orders of magnitudes increased reactions was considerably lower than the usual cases by checking the optical emission from the bremsstrahlung. Exactly this is a proof for of a non-heating plasma block reaction.

This result is also a proof that the laser used in the cases $[15,16,24]$ and the splendid reconfirmation of the measurements [15] with the blue Doppler shift by Földes et al. [26] is proofing that the used lasers lasers 


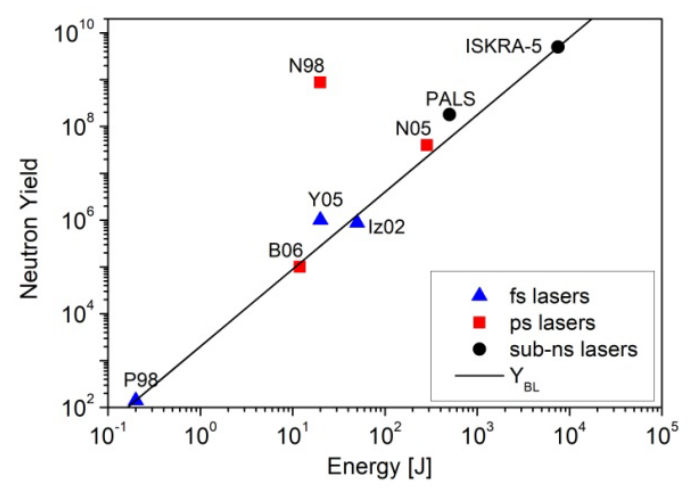

Fig. 4 Compilation of emission of fusion neutron gains from deuterated targets irradiated by laser pulses of varying energy and varying pulse duration [25]. At thermal equilibrium interaction, the results were on a line, but the results with picoseconds pulses N98 with non-thermal block acceleration were increased by a factor 10,000 against the thermal equilibrium cases.

had the perfect high quality of the contrast ratio. Measurements with numerous lasers in other laboratories did not have the sufficiently high quality and could not demonstrate the non-thermal plasma block interaction. Fusion gains at low plasma temperature by block acceleration in the target [27] demonstrated the contrast to the numerous fusion measurements under heating at thermal equilibrium conditions. The same extreme quality is then confirmed in the measurements of the team of Jie Zhang [18] analyzing how to suppress relativistic self-focusing. Fig. 4 indicated this in a drastic way. The measurements [24] N98 and B06 were with same ps laser pulses of same power, however the measurement of Norreyes et al., N98, was with extremely high contrast ratio for producing block generation with the 10,000 times higher fusion gains in contrast to B06 with usual pulses working at thermal equilibrium conditions. In the first case, the 10,000 times increased neutron gains were measured at exceptionally low temperature in contrast to B06 [28]. The measured low temperature of N98 [24] (Fig. 4) confirms the modest emission [27] of bremsstrahlung as needed for the following described HB11 laser fusion generator for electric power. The necessary thermal non-equilibrium was noticed also in a general way [29].

\section{Fusion Reactor for Hydrogen with Boron ${ }^{11} \mathrm{~B}$}

Computations with ps energy deposition on plane solid deuterium-tritium fusion targets resulted in the threshold for ignition needing an energy flux of $10^{8}$ $\mathrm{J} / \mathrm{cm}^{2}$ [20]. These computations were updated [21] and when instead of the DT fusion that of hydrogen $\mathrm{H}$ with the boron isotope 11 (HB fusion) was used, resulted in equal energetic helium (alpha particles)

$$
\mathrm{H}+{ }^{11} \mathrm{~B}=3{ }^{4} \mathrm{He}+8.7 \mathrm{MeV}
$$

The mentioned surprising result turned out [22] to be about on the same threshold E* as DT fusion. This was of interest for a fusion reactor.

The very first HB11 fusion, Eq. (4), in a laboratory was measured by Belyaev et al. [30] resulting in 1,000 reactions per shot, just above the detection threshold. This low level can be understood from Fig. 4 where the used laser was not of the high contrast quality, see B06. When in addition of a ps laser pulse, $\mathrm{MeV}$ ions from a separate laser interaction were incident [31] more than a million reactions were measured. When without these ion beams only one laser pulse was hitting a silicon crystal with very high HB11 concentration [32], more than a billion HB11 reactions were measured. This led to the design of a modestly heated electricity power reactor (Fig. 5 [33]) based on Mourou's co-authorship for using ultra-extreme ps CPA laser pulses with more than $10 \mathrm{PW}$ power. A crucial ingredient was to use nanosecond ultrahigh magnetic kilogauss fields just available by Fujioka at al. [34] from a ns-laser pulse driven capacitor-loop in the center of the rector sphere. The summary of a road map for building a prototype reactor within few years was given [35].

When measurements of HB11 reactions resulted in billion times higher fusion gains [32] first estimations [14] included the three-fold alpha multiplication. Eq. (4) was based on preceding computations [33] using 


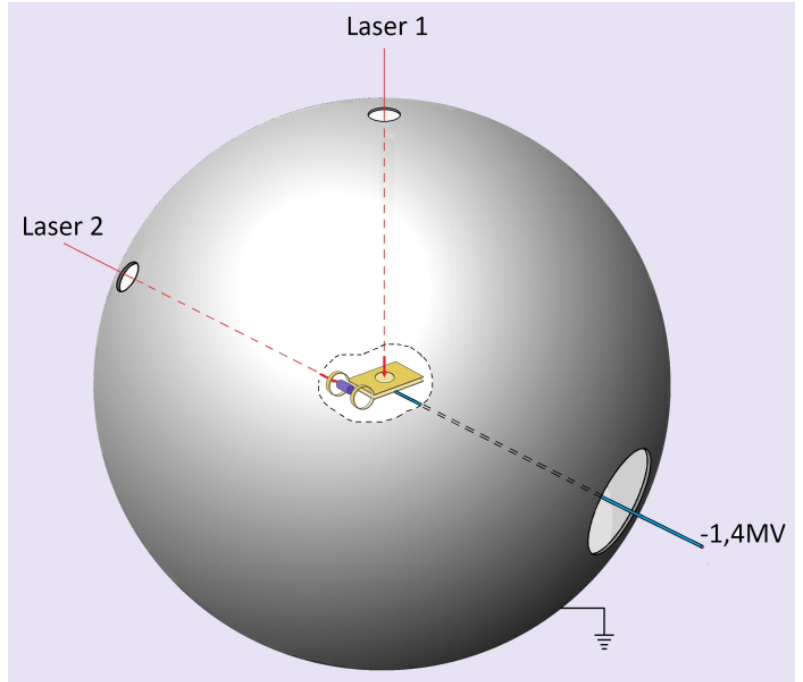

Fig. 5 Scheme of an economic electric power reactor for production of laser-boron-fusion, absolutely free from the problem of dangerous nuclear radiation [33] with the estimated possibility of a power station producing electricity at low costs.

the genuine two fluid hydrodynamics. A detailed analysis of the avalanche reaction by inclusion of the elastic nuclear collisions between the nuclei resulted in an increase of the gains by four orders of magnitudes [36]. Adding this increase by four magnitudes to the five orders from the block mechanism [22] results in the measured billion times higher gains than classical in agreement with the measurements [32].

The design of an environmentally clean, safe, comparably early available boron fusion reactor for low cost electricity was possible [14, 35]. As a consequence, the design of a spherical HB11 fusion reactor was given, in Fig. 5 [33], where the solid density fuel had to be in cylindrical form and the reaction is trapped by the recently produced ultrahigh kilotesla magnetic fields produced [34] by capacitor coils at irradiation of $\mathrm{kJ}$ nanosecond laser pulses. The end-on initiation of the fusion reaction in the cylinder needs picoseconds CPA-laser pulses [19] of more than $30 \mathrm{~kJ}$ energy. These types of lasers are close to be on the market with minute repetition time and shorter producing $277 \mathrm{kWh}$ of electricity per shot from burning 14 milligrams of boron [33, 35]. The measurements of Steinke et al. [37] demonstrated that the non-thermal energy density for the sub-picosecond CPA-laser pulse was much higher compared with thermal fusion pressures at solid state densities. These are directed into the cylinder axis above $\mathrm{MeV}$ energy for the reaction imbedded in a low electron temperature background for space charge neutralizing. The focus cross section is one square millimeter as needed for the conditions of the reactor.

In principle, the solution has been reached, how can fusion energy drive electric power generators without needing thermal pressures with temperatures above 50 million degrees Kelvin instead by using the non-thermal pressures of ultra-extreme ps CPA laser pulses?

The next tasks are the studies of the time dependent properties of the processes of the cylindrical HB11 fusion fuel in the trapped volume of the reactor unit [34] and the repetition of the sub-picosecond interaction of the few dozens of PW power for the direct driven initiation mechanism at the end plane of the solid HB11 fuel volume. The use of plasma block ignition has well been explored by experiments and computer simulations, where a number of details have to be clarified including the highly non-equilibrium sate of the plasma with modest temperature background [38].

\section{Acknowledgements}

This work was supported by the HB11 Energy Pty. Ltd., Freshwater NSW, Australia with most valuable attention by the Advisory Board members Dr. E. Michael Campbell, Director, Laboratory of Laser Energetics, University of Rochester Rocheter NY, USA; Prof. Shalom Eliezer SOREQ Research Center Israel and Polytechnic University Madrid/Spain; Prof George H. Miley Department Nuclear, Plasma \& Radiol. Engineering University of Illinois, Urbana IL, USA; and Prof. Dr.Dr.h.c. Roland Sauerbrey Scientific Director, Helmholtz-Zentrum Dresden-Rossendorf, Germany. 


\section{References}

[1] Grieger, G., and Wendelstein Team. 1981. "Measuremets at the Wendelstein Stellarator. Plasma Physics and Controlled Fusion Research." In Proceedings IAEA Vienna, Vol. 1, pp. 173, 185.

[2] Campbell, E. M. "Foreword" of Edward Teller Lectures, edited by Hora, H., and Miley, G. H., 2nd ed., London: Imperial College Press, p. 1.

[3] Linlor, W. I. 1963. "Ion Energies Produced by Laser Giant Pulse.” Appl. Phys. Letters 3: 210.

[4] Honig, R. E. 1963. "Laser-Induced Emission of Electrons and Positive Ions from Metals and Semiconductors." Appl. Phys. Letters 3 (1): 8-11.

[5] Isenor, N. R. 1964. "Metal Ion Emission Velocity Dependence on Laser Giant Pulse Height." Appl. Phys. Letters 4 (8): 152-3.

[6] Sucov, E. W., Pack, J. L., Phelps, A. V., and Engelhardt, A. G. 1967. "Plasma Production by a High-Power Q-Switched Laser." Physics of Fluids 10: 2035.

[7] Hora, H. 1961. "Experimental Results of Free Targets." In Laser Interaction and Related Plasma Penomena, edited by H. Schwarz and H. Hora. New York: Plenum Press, Vol. 1, 273.

[8] Hora, H. 1981. Physics of Laser Driven Plasmas. New York: Wiley.

[9] Hora, H., Pfirsch, D., and Schlüter, A. 1967. "Acceleration of Inhomogeneous Plasma by Laser Light." Zeitschrift f. Naturforschung 22A: 278.

[10] Hora, H. 1969. "Nonlinear Confining and Deconfining Forces Associated with the Interaction of Laser Radiation with Plasma." Physics of Fluids 12: 182.

[11] Hora, H. 1985. "The Transient Electrodynamic Forces at Laser-Plasma Interaction." Physics of Fluids 28: 3705.

[12] Hora, H. 2010. Laser Plasma Physics, 2nd ed. WA: SPIE Bellingham.

[13] Feynman, R., Section 6.3 of [12].

[14] Strickland, D., and Mourou, G. 1985. "Compression of Amplified Chirped Optical Pulses.” Opt. Comm. 65: 219.

[15] Sauerbrey, R. 1996. "Acceleration of Femtosecond Laser Produced Plasmas." Physics of Plasmas 3: 4712.

[16] Badziak, J., Kozlov, A. A., Makowski, J., Paris, P., Ryz, L., Wolowski, J., et al. 1999. "Investigation of Ion Streams Emitted from Plasma Produced with a High Power Picoseocond Laser." Laser and Part. Beams 17: 323.

[17] Hora, H., Badziak, J., Boody, F. P., Höpfl, R., Jungwirth, K., Kralikowa, B., et al. 2002. "Effects of ps and ns. Laser Pulses for Giant Ion Source." Optics Communications 4: 207.

[18] Zhang, M., He, J. T., Chen, D. B., Li, Z. H., Zhang, Y., Wang, L., et al. 1998. "Effects of a Prepulse on $\gamma$-Ray
Radiation Produced by a Femtosecond Laser with Only 5-mJ Energy." Phys. Rev. E 57: 3745.

[19] Hora, H. 1975. "Theory of Relativistic Self-focusing of Laser Radiation in Plasmas." J. Opt. Soc.of America 65: 882.

[20] Chu, M. S. 1972. "Thermonuclear Reaction Waves at High Densities." Phys. Fluids 15: 412.

[21] Hora, H., Malekynia, B., Ghoranneviss, M., Miley, G. H., and $\mathrm{He}, \mathrm{X}$. 2008. "Twenty Times Lower Ignition Threshold for Laser Driven Fusion Using Collective Effects and the Inhibition Factor." Appl. Phys. Lett. 93: 011101.

[22] Hora, H., Miley, G. H., Azizi, N., Malekynia, B., Ghoranneviss, M., and He, X. T. 2009. "Nonlinear Force Driven Plasma Blocks Igniting Solid Density Hydrogen Boron: Laser Fusion Energy without Radiaoactivity." Laser and Particle Beams 27: 491-6.

[23] Hora, H., and Miley, G. H. 2011. "Possibiity for Gaining Nuclear Energy without Radioactivity from Solid Density Hydrogen Boron Using Laser with Nonlinear Force Driven Plasma Blocks." Journal of Energy and Power Engineering 5: 728 .

[24] Norreys, P. A., Fews, A. P., Beg, F. N., Bell, A. R., Dangor, D. A., Lee, P., et al. 1998. "Neutron Production from Picosecond Laser Irradiation of Deuterated Targets at Intensities of $10^{19} \mathrm{~W} / \mathrm{cm}^{2}$." Plasma Phys. Contr. Fusion 40: 175.

[25] Krasa, J., Klir, D., Velyhan, A., Margarone, D., Krousky, E., Jungwirth, K., et al. 2013. "Observation of Repetive Bursts in Emission of Fast Ions and Neutrons in Sub-nanosecond Laser-Soid Experiments." Laser and Particle Beams 31: 395.

[26] Földes, I., Bakos, J. S., Gal, K., Juhasz, Y., Kedves, M. A., Koscis, G., Szatmari, S., and Verex, G. 2000. "Properties of High Harmonics Generation by UV Laser Pulses on Solid Surfaces." Laser Physics 10: 264.

[27] Krasa, J., Hoffmann, D. H. H., Hora, H., Eliezer, S., Nissim, N., Kirchhoff, J., et al. 2019. "CPA-Laser Pulses for Non-thermal Initiation of Laser-Boron-Fusion." Website HB11 Energy, Publication February 2019.

[28] Belyaev, V. S., Vinogradov, V. I., Kurilov, A. S., Matafonov, A. P., Andrianov, A. P., Ignateev, G. N., et al. 2004. "Neutron Production in a Picosecond Plasma at Radiation Intensity of $3 \times 10^{17} \mathrm{~W} / \mathrm{cm}^{2}$." JETP 98: 1133 .

[29] Fa, Z. F., Lin, Y. Y., Lin, B., Yu, C. X., Lan, K., and Liu, J. 2017. "Thermal Non-equilibrium Conditions in Fusion Plasmas." Matter and Radiation at Extremes 2: 3.

[30] Belyaev, V. S., Matafonov, A. P., Vinogradov, V. I., Krainov, V. P., Lisista, V. S., Roussetski, A. S., et al. 2005. "Observation of Neutron-Less Fusion Reactions in Picosecond Laser Plasmas.” Phys. Rev. 72: 026406.

[31] Labaune, C., Deprierraux, S., Goyon, S., Loisel, C., 


\section{Degrees Temperature for Laser-Boron-Fusion}

Yahia, G., and Rafelski, J. 2013. "Fusion Reactions Initiated by Laser Accelerated Particle Beams in Laser Produced Plasmas." Nature Communications 4: 2506.

[32] Picciotto, A., Margarone, D., Velyhan, A., Bellini, P., Krasa, J., Szydlowski, A., et al. 2014. "Boron-Proton Nuclear-Fusion Enhancement Induced in Boron-Doped Silicon by Low Contrast Pulsed." Phys. Rev. X (4): 031030 .

[33] Hora, H., Korn, G., Giuffrida, L., Margarone, D., Picciotto, A., Krasa, J., Jungwirth, K., Ullschmied, J., Lalousis, P., Eliezer, S., Miley, G. H., Moustaizis, S., and Mourou, G. 2015. "Fusion Energy Using Avalanche Increased Boron Reactions for Block Ignition by Ultrahigh Power Picosecond Laser Pulses." Laser and Particle Beams 33: 607.

[34] Fujioka, S., Zhang, Z., Ishihara, K., Shigemori, K., HironakaI, Y., Johazaki, T., et al. 2013. "Kilotesla Magnetic Field due to a Capacitor Coil Target Driven by
High Power Laser." Nature Sci. Rep. 3: 1170.

[35] Hora, H., Eliezer, S., Kirchhoff, G. J., Nissim, N., Wang, J. X., Lalousis, P., et al. 2017. "Road Map to Clean Energy Using Laser Beam Ignition of Boron-Hydrogen Fusion." Laser and Particle Beams 35: 730.

[36] Eliezer, S., Hora, H., Korn, G., Nissim, N., and Martinez, Val, J. M. 2016. "Avalanche Proton-Boron Fusion Based on Elastic Nuclear Collisions." Physics of Plasmas 23: 050704.

[37] Steinke, J., Henig, A., Schnürer, M., Sokollik, T., Nickles, P. V., Jung, D., et al. 2010. "Efficient Ion Acceleration by Collective Laser-Driven Electro-Dynamics with Ultrathin Foils." Laser and Particle Bemas 28: 215.

[38] Krasa, J., Hoffmann, D. H. H., Hora, H., Eliezer, S., Nissim, N., Kirchhoff, J., et al. 2019. CPA-Laser Pulses for Non-thermal Initiation of Laser Beam Fusion, edited by K. Weyrich and G. H. H. Hoffmann. GSI Report Darmstadt 2019/2. 